www.jmscr.igmpublication.org

Impact Factor 5.84

Index Copernicus Value: 71.58

ISSN (e)-2347-176x ISSN (p) 2455-0450

crossref DOI: _https://dx.doi.org/10.18535/jmscr/v5i11.226

Journal Of Medical Science And Clinical Research

IGM Publication

An Official Publication of IGM Publication

\title{
A Comparative Study of the Therapeutic Effects of Copper Sulpate versus Common Salt (Sodium Chloride) in the Treatment of Infantile Umbilical Granuloma
}

Authors

\section{Dr. Mudassar Fiaz ${ }^{1}$, Dr Adnan Bashir Bhatti MD², Dr. Naveed Ahmed², Dr. Rafee Raza Ahmed ${ }^{2}$}

${ }^{1}$ Assistant Professor, Department of Neonatal \& Paediatric Surgery, Children Hospital, PIMS, Islamabad

${ }^{2}$ Department of Neonatal \& Paediatric Surgery, Children Hospital, PIMS, Islamabad, Pakistan Corresponding Author

Dr Adnan Bashir Bhatti, MD

Department of Neonatal \& Paediatric Surgery, Children Hospital, PIMS, Islamabad, Pakistan

Email:dr.adnanbashir@gmail.com

\begin{abstract}
Background: The most prevalent inflammatory abnormality in the neonates is an umbilical granuloma. It is a benign growth that forms from excessive granulation and failure of epithelialization at the umbilical base. It is not a congenital abnormality and does not present at birth. There is a range of treatment options from silver nitrate, copper sulfate to surgical excision, and cauterization. However, there is a dearth of evidence in the literature concerning the single best treatment option. This study serves that purpose in comparing the therapeutic effects of copper sulfate with the common salt.

Methods: This comparative experimental study was conducted in Pakistan Institute of Medical Sciences (PIMS) for six months using WHO calculator, 60 patients from both genders between ages 2 weeks to 4 months having umbilical granuloma were enrolled. A written informed consent was taken from parents. By lottery method, patients were allocated into group A receiving treatment with copper sulfate; and group B receiving common salt. The outcome was recorded in terms of frequency of excellent response.
\end{abstract}

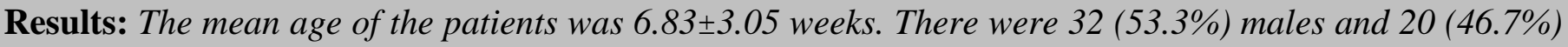
females. No statistically significant difference was observed in the mean age, duration of symptoms, and gender. Significant frequency of excellent response was observed in the copper sulfate group (100\% vs. $53.3 \%$; $p<0.05)$ as compared to the common salt group.

Conclusion: Copper sulfate is a better treatment option than common salt for the treatment of umbilical granuloma.

Keywords: umbilical granuloma, copper sulfate, common salt.

\section{Introduction}

Umbilical granuloma (UG) is a prevalent deformity of the umbilicus in neonates and has been dubbed the most common umbilical abnormality in this group of neonates ${ }^{[1,2]}$. It is a benign abnormality of the neonates that is the result of too much granulation tissue after the cord has separated from the umbilicus ${ }^{[1]}$. It also 
commonly occurs as a reaction to a mild infection ${ }^{[3]}$. Following the infection or cord separation, a tiny portion of a slightly wet, bright red, flesh remains in the umbilical cord. Failure of epithelialization of this left-over granulation tissue leads to the formation of umbilical granuloma ${ }^{[4]}$. Typically, it has a smooth surface morphology although it may sometimes present in a pedunculated manner ${ }^{[5]}$. In the cases of infection, oozing, discharge, and irritation may result if not treated on time ${ }^{[4]}$.

Umbilical Granulomas are typically devoid of sensation because they do not contain any nerves. As a result, they may not cause any pain or noxious sensations to the neonate. However, the presence of mass in place of the umbilical cord that has fallen off may lead to psychological distress in the parents and may indicate therapy ${ }^{[3]}$. Also, the fact that no record of spontaneous resolution of UG has been found in the literature points towards the need for treatment. A number of different treatment modalities have been considered for UG. Some of these include cauterization with silver nitrate (most commonly used), common salt, copper sulfate, cryotherapy, alcohol, and ligation of the granuloma ${ }^{[4,6]}$. However, there is presently no consensus in the literature as regards to a single effective treatment option for the management of UG. For example, a study carried out by Brødsgaard et al. ${ }^{[4]}$ showed that Clobetasol propionate cream is similar in efficacy to topical silver nitrate. On the other hand, Annapurna et al. ${ }^{[6]}$ revealed that copper sulfate is a better treatment option as compared to common salt. Contradicting this was Hossain et al. [7] who concluded that sodium chloride was a better option with almost no side effects and better response rate of $91.7 \%$. Each of these methods has its merits and demerits, and some may have significant side effects, such as reports of skin burns following the use of silver nitrate ${ }^{[3]}$.

The difficulty in arriving at a consensus treatment for UG indicates the need for further studies focused on determining the best treatment option for UG with minimal side effects. Considering that the most commonly used methods are copper sulfate and common salt, it is necessary to compare the therapeutic efficacies of these two treatment options in a randomized controlled trial, hence this study. This study reports the comparative efficacies of copper sulfate and common salt for the treatment of UG.

\section{Materials and Methods}

This comparative experimental study was conducted at the Department of Pediatric Surgery, Children Hospital, PIMS, Islamabad. All requirements of the hospital's ethics committee were fulfilled before starting the study, and a written approval letter was taken. Using WHO calculator, 60 infants (male and female), between the ages of 2 weeks to 4 months were selected. Two groups of infants were decided by a lottery method. Group A received treatment of copper sulpate applied once over the UG for 10 minutes. Group B, was administered with common salt (sodium chloride). A pinch of common salt was applied over the UG for 30 minutes, twice daily for three days. A sterile gauze and adhesive bandage were applied in both groups after salt application. Treatments were administered in outpatient settings, and the patients were followed up at 1 week, 3 weeks, and 1-month interval to record the outcome. Complete regression, complete epithelialization, and no discharge interpreted excellent response while partial regression/epithelialization and no regression represented no response.

The output variable was the frequency of excellent response. The sampling technique utilized was non-probability consecutive sampling. All those infants who met the age range, from both genders and having umbilical granuloma were selected; whereas patients whose parents did not give written consent, with the underlying dermatological condition, with ongoing medical treatment, and with an umbilical hernia were excluded. The study was conducted within 6 months. 


\section{Data Analysis}

The data were analyzed using the Statistical Package for the Social Sciences (SPSS) version 17. Numerical variables such as age, gender, and duration of symptoms were presented as mean + SD. Categorical variables which include gender and frequency of excellent response were recorded in the form of frequency and percentage table (Table 1). Chi-square test was applied to the frequency of excellent response to comparing the two study groups, taking p-value $<0.05$ as significant.

The frequency of excellent response variable was stratified further for age, gender, duration of symptoms to address effect modifiers. After stratification, the chi-square test was applied again to compare the frequency of excellent response among different numerical and categorical variables taking $p$-value of $<0.05$ as significant.

\section{Results}

The age of the selected patients ranged from 2 weeks to 16 weeks with the mean age of
$6.83 \pm 3.05$ weeks. Forty-Six patients $(76.7 \%)$ were aged below 2-months. Thirty-two males (53.3\%), and 28 females $(46.7 \%)$ were enrolled with a male to female ratio of $1.1: 1$. The duration for which the symptoms persisted was between 6 days to 34 days with a mean of $16.33 \pm 7.02$ days.

No statistically significant difference was noted in the two groups in terms of age $(\mathrm{p}=0.802)$, age groups $(\mathrm{p}=0.542)$, duration of symptoms $(\mathrm{p}=0.942)$, and gender $(\mathrm{p}=0.605)$. Therefore, baseline demographic and clinical characteristics were mostly the same across both groups.

A statistically significant difference was noted in the frequency of excellent response between the two groups. In total, 46 patients $(76.7 \%)$ showed excellent response to treatment. Excellent response frequency was significantly higher in Group A patients receiving copper sulfate (100\%) as compared to group B receiving common salt $(53.33 \%) \quad(p=0.00)$. This difference was significant across all ages, in both genders, and symptom duration.

Table 1: Frequency of Excellent Response

\begin{tabular}{|l|c|c|c|}
\hline \multirow{2}{*}{ Characteristics } & \multicolumn{2}{|c|}{ Excellent Response $(\mathrm{n}=46,76.7 \%)$} & \multirow{2}{*}{ P value } \\
\cline { 2 - 4 } & Copper Sulpate $(\mathrm{n}=30)$ & $\begin{array}{c}\text { Common Salt } \\
(\mathrm{n}=30)\end{array}$ & \\
\hline Overall & $30 / 30(100 \%)$ & $16 / 30(53.3 \%)$ & $0.000^{*}$ \\
\hline Age Groups & $24 / 24(100 \%)$ & $12 / 22(54.5 \%)$ & $0.000^{*}$ \\
\hline$<2$ months & $6 / 6(100 \%)$ & $4 / 8(50.0 \%)$ & $0.040^{*}$ \\
\hline 2-4 months & $17 / 17(100 \%)$ & & \\
\hline Gender & $13 / 13(100 \%)$ & $8 / 15(53.3 \%)$ & $0.001^{*}$ \\
\hline Male & & & $0.004^{*}$ \\
\hline Female & $24 / 24(100 \%)$ & $13 / 24(54.2 \%)$ & $0.000^{*}$ \\
\hline Duration Groups & $6 / 6(100 \%)$ & $3 / 6(50.0 \%)$ & $0.046^{*}$ \\
\hline 6-20 days & \multicolumn{2}{l}{} \\
\hline 21-34 days & \multicolumn{2}{|c|}{} & \\
\hline *Chi square test; observed difference was statistically significant & & \\
\hline
\end{tabular}

\section{Discussion}

An umbilical granuloma (UG) is the most prevalent pediatric abnormality which is a consequence of failed epithelialization and increase growth of granulation tissue at the umbilicus after separation of umbilical cord ${ }^{[1,4]}$. Because of its high prevalence, they are commonly seen in the pediatric clinics. If left untreated, or not properly treated, they may become complicated and led to the formation of pus for several months ${ }^{[8]}$. Umbilical Granulomas have also been reported to lead to the development of necrotizing fasciitis and omphalitis $^{[9]}$. In the literature, there are presently no records of spontaneous resolution in children with umbilical granuloma, so treatment is 
necessary ${ }^{[10]}$. There are different treatment options which have been propounded such as chemical cauterization, electrical cauterization, cryocauterization, and treatment with topical copper sulfate, and common salt ${ }^{[6]}$. Small granulomas can be treated with silver nitrate remedy while larger ones may require surgical resection ${ }^{[5]}$. In the past, cauterization, with $75 \%$ silver nitrate was the most commonly used treatment ${ }^{[7]}$. The use of silver nitrate has been drastically cut down due to its propensity to cause burns if not well administered ${ }^{[3]}$. On the other hand, the use of topical antibiotics in treating UG has not been successful so far, which indicates that UG is not purely a bacterial infection $^{[11]}$. However, Wang et al. ${ }^{[9]}$ researched topical doxycycline for umbilical granuloma and concluded it to be a safe drug with high efficacy. Nonetheless, every treatment modality has its own merits and demerits, and the need for this current study arose from Brodsgaard et al. who mentioned the lack of evidence for an efficacious treatment modality for umbilical granuloma ${ }^{[4]}$.

This comparative study compared the therapeutic effects of topical copper sulfate and common salt. In our study, the majority of the patients with UG were under the age of 2 months. This is consistent with the study of Anapurna et al. ${ }^{[6]}$ and Saleh et al. ${ }^{[3]}$ who reported the prevalent age group is less than two months in $82.14 \%$ and $72.0 \%$ respectively. A study by Assi et al. showed that out of 125 study participants, 86 were aged less than 2 months of age which is also consistent with our study ${ }^{[12]}$. It is therefore clear that children under the age of two months suffer more from UG than older children. It is not surprising as UG is related to the umbilical cord, and which falls off a few days after birth. The presence of UG, if present, is detected immediately after that, and necessary corrective steps should be taken to treat it. Also, our study showed a slight predominance of the male gender, 1.1:1. Similar findings were found by Anapurna et al. ${ }^{[6]}$. However, a contrary finding was seen in the study of Saleh et al. who reported a higher prevalence of UG in the female gender $^{[3]}$. The mean age, duration of symptoms, and gender distribution were not significantly different between the two study groups.

Based on the outcomes of the frequency of excellent response, it was observed that all the 30 patients $(100 \%)$ of the group A receiving copper sulfate had an excellent response to the treatment. On the other hand, Group B receiving common salt, only $16(53.3 \%)$ showed excellent response outcome. There was a significant difference in the response of both treatments $(\mathrm{p}=0.00)$. This difference was significant across all age groups, both genders, and duration of symptoms. This finding is consistent with the observations of Annapurna et al. $^{[6]}$ who found an excellent response with copper sulfate group $(95.5 \%)$ and slightly better response to the common salt group (55\%). This suggests that copper sulfate may be a more efficacious form of therapy than the common salt. On the other hand, while working on therapeutic effects of common salt in the treatment of UG, Hossain et al. ${ }^{[7]}$ showed $91.67 \%$ excellent response rate to the common salt treatment. This suggests that common salt cannot afford to be jettisoned, but raises the questions of why Hossain et al. found a higher response to treatment with salt, while our present study, as well as Annapurna et al. ${ }^{[6]}$ found a relatively lower response to the treatment. It is possible that some conditions that appear to be UG might have been misdiagnosed, as Hossain et al. suggest that diagnostic errors occur and this may delay the initiation of the proper treatment. However, as far as our study is concerned, no specific factors accounting for the low therapeutic efficacy of common salt are apparent at the time of the study, but suggest the need for further studies to elucidate why the use of common salt has been reported with different treatment efficacies in different studies.

From the results of this study, copper sulfate has a higher treatment efficacy and can be recommended as the first line of treatment for UG. However, in consideration of other factors such as availability, affordability, and accessibility, the use of the common salt as a treatment option for 
UG cannot be dismissed. Common salt is more readily available, less costly, but less effective in its therapeutic efficacy when compared to copper sulfate for the treatment of UG. Also, another advantage of common salt is its much-reduced tendency to cause side effects. Its therapeutic effects arise through its desiccant properties, as the high concentration of sodium ions draws water out of the UG and causes it to shrink and necrose. Since these effects are not strong enough to affect surrounding skin, it becomes easier to administer, even by parents or non-medical individuals. However, for the purpose of more efficacious therapies, copper sulpate, in the light of present evidence, is a better option than the common salt.

\section{Conclusion}

From the findings of this study, it is concluded that copper sulfate has better therapeutic outcomes when compared with the common salt for the treatment of umbilical granuloma. More infants with UG stand a higher and approximately 100 percent chance of having an excellent response to treatment with the use of copper sulfate. However, the common salt, even though not as effective as copper sulfate, is another important therapeutic option for UG due to its simple, easy availability, inexpensive, and moderately effective nature without the risk of complications or side effects.

\section{Conflict of Interest}

The author finds no conflict of interest.

\section{References}

1. Alexander $G$, Walsh $R$, Nielsen A. Neonatal umbilical mass. West J EmergMed2013;14(2):163.doi: 10.5811/westjem.2012.8.13249.

2. O'Donnell KA, Glick PL, Caty MG. Pediatric umbilical problems. PediatrClin North Am 1998;45:79199.doi:10.1016/S0031-3955(05)70045-6.

3. Saleh AA. Therapeutic effect of common salt on umbilical granuloma in infants. Int
J Med Sci Pub Health2016;5(5):911-4.doi: 10.5455/ijmsph.2016.07012016312.

4. Brodsgaard A, Nielsen T, Molgaard U, Pryds O, Pedersen P. Treating umbilical granuloma with topical clobetasol propionate cream at home is as effective as treating it with topical silver nitrate in the clinic. ActaPaediatr 2015;104:174-7.doi: 10.1111/apa.12824.

5. Disorders of the umbilicus in infants and children: A consensus statement of the Canadian Association of Paediatric Surgeons. Paediatrics\& Child Health. 2001;6:312-313.doi: 10.1093/pch/6.6.312.

6. Annapurna D, Ramu P. Therapeutic effect of copper sulfate vs common salt (table/cooking salt) on umbilical granuloma in infants: a comparative study. J Evol Med Dent Sci2015;4:1616-21. doi: 10.14260/jemds/2015/228.

7. Hossain AKMZ, Hasan GZ, Islam KMD.Therapeutic effect of common salt (table/ cooking salt) on umbilical granuloma in infants. Bangladesh J Child Health 2010;34:99-102. doi: 10.3329/bjch.v34i3.10360.

8. Nagar H. Umbilical Granuloma: a new approach to an old problem. Pediatr SurgInt2001;17:513-4. doi: 10.1007/s003830100584.

9. Wang, Huaijie et al. Dramatic response of topical doxycycline in umbilical granuloma. Global Pediatric Health 2015:2333794X1560731.doi: $10.1177 / 2333794 X 15607315$.

10. Snyder, Charles L. Current management of umbilical abnormalities and related anomalies. Seminars in Pediatric Surgery 2007: 41-49. doi: 10.1053/j.sempedsurg.2006.10.006.

11. Wooltorton. Noninvasive treatments for umbilical granulomas. American Family Physicians 2003;67:698-700 <http://www.aafp.org/afp/2003/0215/p698 .html> 
12. Assi AN, Kadem MK, Rubaee RJ, Atshan FG. Management of umbilical granuloma. Thi-Qar Medical Journal 2010;4:82-87 $<$ http://www.iasj.net/iasj?func=fulltext\&aI $\mathrm{d}=49280>$ 